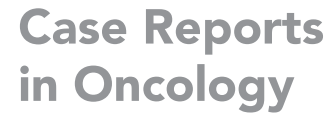

\title{
A Case of Breast Cancer in a Patient with a Congenital Pectoralis Muscle Defect
}

\author{
Tsuyoshi Nakagawa ${ }^{a}$ b Goshi Oda ${ }^{a}$ Rie Kato $^{b}$ Hajime Shinohara \\ Takayuki Osanai $^{b}$ Yuichi Kumaki ${ }^{a}$ b Noriaki Takiguchi ${ }^{b}$ Hiroki Mori ${ }^{c}$ \\ Jun Oyama ${ }^{d}$ Kentaro Okamoto ${ }^{e}$ Hiroyuki Uetake ${ }^{e}$ \\ ${ }^{a}$ Department of Breast Surgery, Tokyo Medical and Dental University, Tokyo, Japan; \\ ${ }^{b}$ Department of Surgery, Tsuchiura Kyodo General Hospital, Ibaraki, Japan; 'Department \\ of Plastic Surgery, Tokyo Medical and Dental University, Tokyo, Japan; ${ }^{\mathrm{d} D e p a r t m e n t}$ of \\ Radiology, Tokyo Medical and Dental University, Tokyo, Japan; 'Department of Specialized \\ Surgeries, Graduated School, Tokyo Medical and Dental University, Tokyo, Japan
}

\section{Keywords}

Breast cancer - Mastectomy · Congenital pectoralis muscle defect

\begin{abstract}
Congenital pectoral muscle defects are very rare, and when accompanied by limb defects, they are called Poland syndrome. A woman in her 70s, 4 years after partial mastectomy for breast cancer, underwent mastectomy for a local recurrence. During the operation, the pectoralis major and minor muscles were found to be defective. However, the patient did not have any limb defects. Although congenital pectoral muscle defects are very rare, it would be better to confirm defects of the pectoral muscle by preoperative diagnostic imaging such as CT because the postoperative treatment may be affected.
\end{abstract}

\section{Introduction}

Congenital pectoralis defects are congenital malformations that occur in about 1 in 20,000 people, and when they are associated with limb loss, they are called Poland syndrome $[1,2]$. In the treatment of breast cancer, defects in the pectoralis major and minor muscle can have a significant impact on surgery and radiation therapy. An extremely rare case is reported, along with consideration of the implications with respect to before and after surgery for breast cancer on the affected side. 
Fig. 1. The left breast is removed, but the pectoralis major and minor muscles are barely present. As noted by the arrow, only a small portion of the pectoralis major muscle is present on the cephalad side.

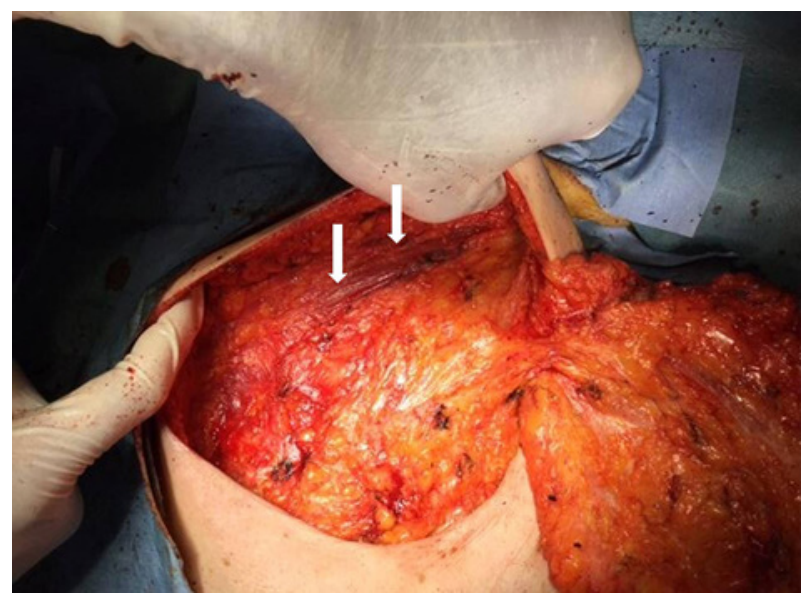

Fig. 2. CT shows that the right pectoralis minor muscle is slightly present on the cephalad side, whereas the left pectoralis major muscle is almost completely absent, and the pectoralis minor muscle is deficient.

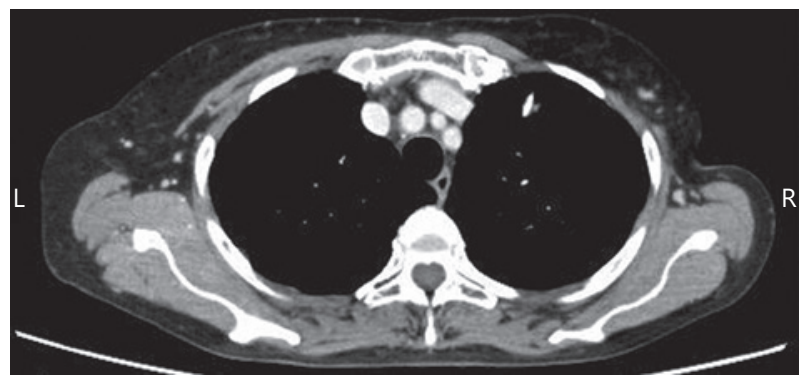

\section{Case Report}

A 70-year-old woman came to the hospital after becoming aware of a breast tumor in the left $\mathrm{C}^{\prime}$ region. A 4-cm mass was found in the left " $\mathrm{C}$ " area, and a needle biopsy from there showed a diagnosis of invasive breast cancer. Partial mastectomy and axillary lymph node dissection were performed. During this surgery, the pectoral muscle defect was unnoticed because the surgery was performed in the axillary region. There was also the possibility of accessory breast cancer, but the final pathological results showed a diagnosis of invasive lobular carcinoma ER (-) PgR (-) HER2:2+ (fluorescence in situ hybridization: negative) and axillary lymph node metastasis. Postoperative adjuvant chemotherapy was planned, and 1 course of epirubicin $100 \mathrm{mg} / \mathrm{m}^{2}$, cyclophosphamide $500 \mathrm{mg} / \mathrm{m}^{2}$, and fluorouracil 500 $\mathrm{mg} / \mathrm{m}^{2}$ was administered, but the side effects were so severe that all adjuvant therapies were discontinued.

Four years after the surgery, she became aware of a left breast tumor and was diagnosed with invasive lobular carcinoma (ER [+] PgR [+] HER2:1) by needle biopsy; local recurrence was diagnosed, and she underwent a total mastectomy. At surgery, after the skin flap was made, the left breast was raised laterally and resected, but the pectoralis major muscle was almost completely absent, with only a small amount present near the clavicle, and the pectoralis minor muscle was completely absent (Fig. 1). This was confirmed by CT. The right pectoral muscle was smaller than normal, but both pectoralis major and pectoralis minor muscles were present (Fig. 2, 3). Reviewing the mammography again, the left mediolateral oblique view did not show the pectoralis major muscle (Fig. 4). The patient's postoperative course was uneventful. The pathological result was invasive lobular carcinoma. Postoperative adjuvant chemotherapy was epirubicin $60 \mathrm{mg} / \mathrm{m}^{2}$ and cyclophosphamide $600 \mathrm{mg} / \mathrm{m}^{2}$ 
Fig. 3. CT shows that there is no pectoralis major or minor muscle dorsal to the left breast cancer. The right pectoralis major muscle is also absent at this height.

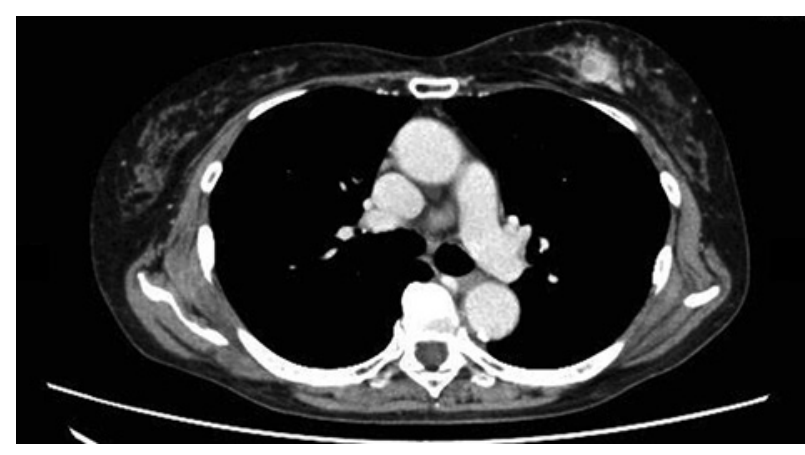

Fig. 4. The left pectoralis muscle is not shown on the mediolateral oblique view of mammography.

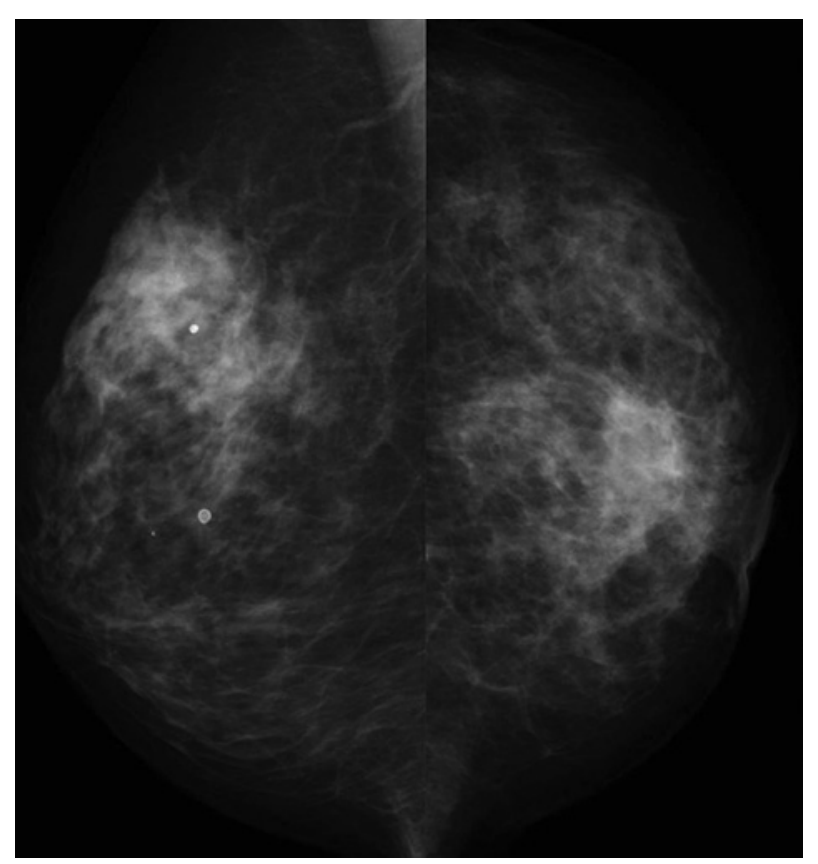

(EC), 4 cycles, every 3 weeks, followed by docetaxel $\left(75 \mathrm{mg} / \mathrm{m}^{2}, 4\right.$ cycles, every 3 weeks). Endocrine therapy was administered with an aromatase inhibitor. Two years have passed since the mastectomy, and no recurrence has been observed. Based on the diagnostic criteria for Poland's syndrome, it was clear that the patient had no limb deficits, and so it was concluded that the patient had a simple pectoralis minor muscle defect.

\section{Discussion}

Congenital aplasia of the pectoralis muscle accounts for $47 \%$ of all congenital aplasia of muscles, with a frequency of 1 in 22,189 [1]. In particular, those with ipsilateral short finger syndrome and syndactyly are considered to have Poland syndrome, with a frequency of 1 in $36,000-50,000$ [3]. The etiology of this condition is still unknown, but the general hypothesis is that it is related to a disruption in the distribution of blood flow through the subclavian axis during the critical sixth week of embryonic development [4]. Although there are no reports suggesting an association with breast cancer, an association with malignant disease such as leukemia has been suggested [5]. 
DeFazio et al. [6] reported that there have been 18 reports of breast cancer cases with Poland syndrome. Interestingly, Poland syndrome is diagnosed incidentally in $63 \%$ of patients, either during the examination or during treatment for breast cancer [6]. Breast cancers that developed in Poland syndrome did not differ from normal breast cancers with respect to the histopathological diagnosis, but they had a higher frequency of lymph node metastasis (31\%) [6]. Therefore, postmastectomy radiotherapy may be necessary, but there is concern that the loss of pectoral muscles may reduce the protective function of the thorax and decrease cardiopulmonary function [7].

When performing a mastectomy for a patient with a pectoral muscle defect, the following 2 points should be considered: (1) the possibility that the perforating branches to the mammary gland are more developed than usual and (2) the possibility that the subclavian vein may be exposed to the surgical field [8]. In this case, the subclavian vein was not exposed due to the presence of the pectoralis major muscle at the clavicle. The mastectomy was completed safely because there was no overdevelopment of the perforating branches.

In the present case, the patient had no desire for immediate breast reconstruction. Autologous tissue reconstruction can be performed with latissimus dorsi flap or deep inferior epigastric perforators flap. Fixation of the skin flap to the anterior thoracic region is possible by suturing it to the periosteum of the clavicle or ribs. On the other hand, breast reconstruction with tissue expander implantation usually involves the placement of a tissue expander in the dorsal side of the pectoralis major muscle, which cannot be placed in patients with a defective pectoralis muscle, such as the present case, and autologous tissue reconstruction should be performed. In addition, direct subcutaneous implant placement should not be done because of the risk of skin ulceration. In the present case, the pectoral muscle defect was not noted before the surgery, and a simple mastectomy was performed without any problems. If immediate artificial breast reconstruction had been planned, the reconstruction procedure would have had to be changed. Therefore, if artificial breast reconstruction were to be performed, it would be necessary to confirm the presence of the pectoral muscle on CT, although pectoral muscle defects are very rare.

\section{Conclusion}

Congenital defects of the pectoral muscle are very rare, but they can affect treatment decisions for breast cancer. The presence of the pectoral muscle should be confirmed by preoperative diagnostic imaging, such as CT.

\section{Statement of Ethics}

All procedures were conducted in accordance with the ethical standards of the Helsinki Declaration of 1975. Patient anonymity and all confidential information have been preserved. Written informed consent was obtained from the patient for publication of this case report and any accompanying images.

\section{Conflict of Interest Statement}

The authors declare that they have no conflicts of interest.

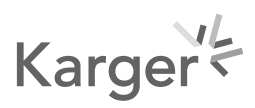




\section{Case Reports in Oncology}

Case Rep Oncol 2021;14:1092-1096

\begin{tabular}{l|l}
\hline DOI: $10.1159 / 000516189$ & ○ 2021 The Author(s). Published by S. Karger AG, Basel \\
\hline
\end{tabular} www.karger.com/cro

Nakagawa et al.: Breast Cancer Patient with a Congenital Pectoralis Muscle Defect

\section{Funding Sources}

This research did not receive any specific grant from funding agencies in the public, commercial, or not-for-profit sectors.

\section{Author Contributions}

T.N. contributed to the writing of the manuscript. G.O., H.M., and H.U. supervised the study. T.N., R.K., H.S., T.O., Y.K., N.T., and J.O. served as the attending physicians for the presented patient. All the authors have read and approved that final version of this manuscript.

\section{References}

1 Castilla EE, Paz JE, Orioli IM. Pectoralis major muscle defect and Poland complex. Am J Med Genet. 1979;4(3): 263-9.

2 Poland A. Deficiency of the pectoral muscles. Guys Hosp Rep. 1841;6:191-3.

3 Ravitch MM. Poland's syndrome-a study of an eponym. Plast Reconstr Surg. 1977 Apr;59(4):508-12.

4 Bavinck JN, Weaver DD. Subclavian artery supply disruption sequence: hypothesis of a vascular etiology for Poland, Klippel-Feil, and Mobius anomalies. Am J Med Genet. 1986 Apr;23(4):903-18.

5 Hoefnagel D, Rozycki A, Wurster-Hill D, Stern P, Gregory D. Leukaemia and Poland's syndrome. Lancet. 1972 Nov 11;2(7785):1038-9.

6 Michael VD, Ornela AD, Laura MB, Troy AP, Michael JO, Eleni AT, et al. Delayed recurrent and bilateral breast cancer in patients with partial Poland's anomaly: report of 2 rare cases and review of the literature. Clin Breast Cancer. 2018 Jun;18(3):e285-e290.

7 Lucas C, Youlia MK, Francois C, Virginie F, Remy JS. Poland syndrome, breast cancer: the importance of the radiotherapy technique after mastectomy. Radiother Oncol. 2009 Apr;91(1):138-9.

8 Yuji O, Masami M, Hiroshi T, Ichiro H, Hiroshi K, Moriyuki N. A case of breast cancer developed on the affected side of the congenital aplasia of the pectoralis muscle. J Jpn Surg Ass. 2020 Aug;81(8):1476-81.

\section{Karger' ${ }^{\prime}$}

